\title{
Bertalanffy-Funktion und Ford-Walford-Formel
}

\author{
FRIEDRICH KRÜGER \\ Biologische Anstalt Helgoland, Zentrale, Hamburg 50
}

\begin{abstract}
Bertalanffy-function and Ford-Walford-formula. Hohendorf (1966) has attempted to apply the relation between the parameters of the FORD-WALFORD-plot and the BERTALANFFY-function, formulated by TAYLOR, to the somewhat difficult calculation of the parameters of the latter function. Working with values, calculated in this manner, demonstrated that they are not mathematically optimal. A careful analysis of the ForDWALForD-formula revealed, that it contains a third parameter which allows, in a simple manner, the derivation of the BERTALANFFY-function. The relation between the parameters of the two functions is complicated by the fact that the regression line of the FOrD-WALFORDformula is adjusted to the minimum of the linear differences between observed and calculated values, while the optimal description of growth requires the minimum of the percentual deviations.
\end{abstract}

\section{EINLEITUNG}

In der Fischerei-Biologie hat man sich sehr intensiv mit der Möglichkeit einer mathematischen Beschreibung von Wachstumsvorgängen befaßt. Das unbegrenzte und nach der larvalen Entwicklung anscheinend durch keine Unstetigkeit komplizierte Wachstum der Fische läßt es als besonders gut geeignetes Beispiel für das Studium von Wachstumsvorgängen erscheinen. Auf die Vielzahl der vorgeschlagenen Funktionen möchte ich hier nicht eingehen und mich vielmehr auf das Problem der Beziehung zwischen der FORD-WALFORD-Darstellung und der BERTALANFFY-Funktion beschränken. Beide Funktionen haben sich als sehr gut brauchbar erwiesen und sind auch in weiterem Umfang angewandt worden. Die weitgehende Ubereinstimmung der zahlenmäßigen Ergebnisse hat BEverton \& HOLT (1957) sowie TAYLOR (1958) veranlaßt, die mathematische Beziehung zwischen beiden Funktionen zu klären.

Die von FORD (1933) und später von WALFORD (1949) aufgedeckte Konstanz der Wachstumsraten in aufeinanderfolgenden Jahren beruht auf einer einfachen Darstellung der Längenwerte, auf deren Wiedergabe dieses Verfahren beschränkt ist. Man ordnet hierbei auf normalem Millimeterpapier den auf der Abszisse abgetragenen einzelnen Längenwerten jeweils den nächst höheren Längenwert als Ordinate zu. Es ergibt sich, daß die auf diese Weise bestimmten Koordinatenpunkte zumeist in sehr guter Näherung auf einer Geraden liegen. Mathematisch liegt dieser Beziehung nach FORD (1933) die Funktion zugrunde:

$$
l_{n+1}=a+b \cdot l_{n}
$$


wenn $l$ die Länge darstellt und $n$ das Alter in Jahren. In der Bezeichnung der Parameter $a$ und $b$ folge ich der Formulierung von HoHendorf (1966).

Die Anwendung dieser Wachstumsdarstellung ist nicht auf das Fischwachstum beschränkt, sondern kann zum Beispiel auch auf das Säugetierwachstum angewandt werden, wie schon WALFORD (1949) gezeigt hat.

Will man Gewichtsdaten auswerten, so muß man aus ihnen die dritte Wurzel ziehen und diese Gewichtslängen $[\sqrt[3]{w}]$ in die Darstellung oder Berechnung einsetzen. Diese Gewichtslängen geben nicht die wirkliche Länge wieder, können aber durch einen geeigneten Faktor in Längenwerte umgewandelt werden. Das Verfahren fordert allerdings, daß ein isometrisches Wachstum vorliegt, was im allgemeinen auch in guter Näherung zutriff. Die FORD-W ALFORD-Darstellung setzt Meßreihen mit konstanten Altersabständen voraus. GulLAND \& Holt (1960) haben das Vorgehen bei ungleichmäßigen Zeitabständen beschrieben.

Auch die BertalanfFy-Funktion:

$$
y_{\tau}=L_{\infty} \cdot\left(1-e^{-K\left(\tau-\tau_{0}\right)}\right)
$$

gestattet nur die Darstellung von Längenwerten. Gewichtswerte müssen - wie bei der FORD-WALFORD-Darstellung - in Form von Gewichtslängen eingesetzt werden. Bei dieser Funktion bereitet die Parameterberechnung gewisse Schwierigkeiten. Aus diesem Grunde versuchten Beverton \& Hol'T (1957) den entscheidenden Parameter dieser Funktion - Lo - auf dem Umweg über die Walford-Darstellung zu ermitteln. Das von ihnen vorgeschlagene graphische Verfahren liefert seiner Natur nach nur Näherungswerte. Günstiger ist der schon von TAYLOR (1958) vorgeschlagene Weg $L_{\infty}$ auf Grund der Beziehung

$$
L_{\infty}=\frac{a}{1-b}
$$

aus der Ford-WaLford-Formel zu errechnen. Man erhält auf diese Weise den Maximalwert dieser Funktion. Für den Parameter $K$ der Bertalanffy-Funktion stellte Taylor die Beziehung auf:

$$
b=e^{-K}
$$

In neuester Zeit hat nun HoHENDORf die von TAYLOR (er zitiert diesen Autor nicht) postulierte Beziehung $z$ wischen den Parametern der beiden Funktionen eingehender zu belegen versucht und auf Grund dieser Werte einige Beispiele durchgerechnet. An einem Beispiel hat er sein Vorgehen näher erläutert und dadurch auch den mathematisch weniger versierten Biologen zugängig gemacht.

Er geht hierbei von der Berechnung der Regressionsgeraden der FORD-WALFORDBeziehung aus, deren Parameter nach seinem Vorschlag ohne Anwendung von Funktionstabellen verhältnismäßig einfach exakt berechnet werden können. Die ForDWALFORD-Parameter rechnet er dann nach den Gleichungen (3) und (4) zu den BERTALANFFY-Parametern um. 


\section{RECHNERISCHE AUSWERT'UNG}

Zur ersten Testung des von HoHENDORF vorgeschlagenen Verfahrens benutzte ich ein von v. BERTALANFFY (1934) durchgerechnetes Beispiel, nämlich die Zahlenreihe von DERJAVIN (1922) für das Längenwachstum des männlichen Störs (Acipenser stellatus). Hierbei ergab sich, daß die von v. Bertalanfry eingesetzten Parameter eine wesentlich bessere Wiedergabe liefern als die nach HoHendorf errechneten Parameter (Tab. 1). Offensichtlich erfült also die von HoHENDORF vorgeschlagene Parameter-Bestimmung nicht die Forderung nach einer mathematisch optimalen Lösung.

Tabelle 1

Vergleich der nach dem Verfahren von HoHENDoRf (1966) berechneten Wachstumswerte von Acipenser stellatus $(\delta)$ mit den Ergebnissen von v. BERTALANFFY (1934)

\begin{tabular}{|c|c|c|c|c|c|}
\hline $\begin{array}{l}\text { Alter } \\
\text { (Jahre) }\end{array}$ & $\begin{array}{c}\text { Meßwerte } \\
(\mathrm{cm})\end{array}$ & $\begin{array}{l}\text { Werte von } \\
\text { V. BERTALANFFY }\end{array}$ & $\begin{array}{c}0 / 0 \\
\text { Abweichung }\end{array}$ & $\begin{array}{l}\text { Werte nach } \\
\text { HoHENDORF }\end{array}$ & $\begin{array}{c}0 / 0 \\
\text { Abweichung }\end{array}$ \\
\hline & & $\begin{array}{l}L_{0}=21,10 \\
L=201,1 \\
K=0,06\end{array}$ & & $\begin{array}{l}L_{O}=20,3 \\
L=209,6 \\
K=0,056\end{array}$ & \\
\hline 1 & 21,1 & 21,1 & $\pm 0,00$ & 20,3 & $-3,79$ \\
\hline 2 & 32,0 & 30,6 & $-4,38$ & 30,6 & $-4,38$ \\
\hline 3 & 42,3 & 41,5 & $-1,89$ & 40,4 & $-4,49$ \\
\hline 4 & 51,4 & 50,8 & $-1,17$ & 49,6 & $-3,50$ \\
\hline 5 & 60,1 & 59,5 & $-1,00$ & 58,3 & $-2,99$ \\
\hline 6 & 68,0 & 67,8 & $-0,29$ & 66,7 & $-1,91$ \\
\hline 7 & 75,3 & 75,5 & $+0,27$ & 74,5 & $-1,06$ \\
\hline 8 & 82,3 & 82,8 & $+0,61$ & 81,9 & $-0,49$ \\
\hline 9 & 89,0 & 89,7 & $+0,79$ & 88,8 & $-0,22$ \\
\hline 10 & 95,3 & 96,2 & $+1,10$ & 95,4 & $+0,11$ \\
\hline 11 & 101,6 & 102,3 & $+0,69$ & 101,6 & $\pm 0,00$ \\
\hline 12 & 107,6 & 108,1 & $+0,46$ & 107,5 & $-0,09$ \\
\hline 13 & 112,7 & 113,5 & $+0,71$ & 113,2 & $+0,44$ \\
\hline 14 & 117,7 & 118,6 & $+0,62$ & 118,4 & $+0,59$ \\
\hline 15 & 122,2 & 123,4 & $+1,00$ & 123,4 & $+1,00$ \\
\hline 16 & 126,5 & 127,9 & $+1,11$ & 128,1 & $+1,26$ \\
\hline 17 & 130,9 & 132,2 & $+0,99$ & 132,5 & $+1,22$ \\
\hline 18 & 135,3 & 136,2 & $+0,67$ & 136,7 & $+1,03$ \\
\hline 19 & 140,2 & 140,0 & $-0,14$ & 140,7 & $+0,36$ \\
\hline 20 & 145,0 & 143,5 & $-1,03$ & 144,5 & $-0,34$ \\
\hline 21 & 148,6 & 146,9 & $-1,14$ & 148,1 & $-0,34$ \\
\hline \multirow[t]{2}{*}{22} & 152,0 & 150,0 & $-1,32$ & 151,4 & $-0,39$ \\
\hline & & \multicolumn{2}{|c|}{$s_{D}= \pm 1,39 \%$} & \multicolumn{2}{|c|}{$s_{D}= \pm 2,13 \%$} \\
\hline
\end{tabular}

Man kann das auch sehr gut an dem von HoHENDorf berechneten Beispiel des Ostsee-Steinbutts (Scophtalmus maximus) demonstrieren. Die von ihm benutzten Daten für das Längenwachstum dieses Fisches lassen sich sehr gut nach dem Ford-WALFORD-Verfahren darstellen. Einen wesentlich besseren Anschluß an die gegebenen Daten erhält man aber, wenn man für den Ausgangswert $\left(l_{l}\right) 10,5 \mathrm{~cm}$ wählt an Stelle des Meßwertes von $10,6 \mathrm{~cm}$. In diesem Falle berechnet sich die Standardabweichung $s_{D}=1,52 \%$. Aus den Werten von Hohendorf ergibt sich dagegen 2,44\%. (Mein 
Wert für $s_{D}$ liegt aus dem Grunde etwas höher als der von HoHendorf errechnete, da ich abweichend von seinem Vorgehen bei der Berechnung 3 Freiheitsgrade abziehe.)

Bei der engen Beziehung $z$ wischen BERTALANFFY-Funktion und WAIFord-Formel war aber anzunehmen, daß auch durch sie eine bessere Wiedergabe der Meßdaten möglich sei, als sie Hohendorf erreichte. Aus diesem Grunde habe ich zur Kontrolle die von v. BertalanfFy (1934) beschriebene graphische Methode zur Bestimmung von $L_{\infty}$ angewandt, wobei ich die nach der WALFORD-Berechnung ausgeglichenen Werte zugrunde legte. Hierbei hatte ich den Eindruck, als ob ein etwas höherer Wert als $33,3 \mathrm{~cm}$ günstiger sei und wählte als Grundlage für die weiteren Berechnungen $33,5 \mathrm{~cm}$. Man kann den hierzu gehörigen Wert für a nach Gleichung (3) berechnen und in die WALFORD-Formel einsetzen. Mit den auf diese Weise gefundenen Werten kann man dann die Güte der Wiedergabe prüfen. Ich variierte $L_{\infty}$ in Stufen von $0,1 \mathrm{~cm}$, und auch hierbei erwies sich rechnerisch 33,50 für $L_{\infty}$ als optimal, das heißt, es ergab sich eine minimale quadratische Abweichung. Diese Berechnungen erfolgten auf der Basis des $b$-Wertes 0,7682 , wie er sich aus der Berechnung von HoHENDorf ergab. Ich habe ihn nicht mehr variiert, da ein solches Vorgehen einen sehr hohen Rechenaufwand erfordert, der nur mit Hilfe eines Computers rationell durchgeführt werden kann. Eine Programmierung wäre aber für den vorliegenden Einzelfall wenig sinnvoll gewesen.

Für $\tau_{0}$ schlägt HoHENDORf eine Methode vor, die mathematisch zwar richtig ist, aber aus dem Grunde zu unbefriedigenden Werten führt, weil sie durch abweichende Meßwerte beeinflußt wird und exakte Werte für die beiden Parameter voraussetzt. Auf dem Wege über $L_{a}$, dessen Wert mit Kenntnis von $l_{f}$, $a$ und $b$ leicht zu errechnen ist, ermittelte ich nach der Formel:

$$
\tau_{0}=\frac{\ln L_{\infty}-\ln \left(L_{\infty}-L_{0}\right)}{K}
$$

$\tau_{\theta}$ zu 0,426. Für die praktische Arbeit an der Rechenmaschine ziehe ich übrigens $L_{\theta}$ als dritten Parameter vor. Der Wert von $\tau_{0}$ erwies sich bei Berechnungen als sehr kritisch.

Die von mir auf diesem Wege erhaltenen BerTalanfFy-Parameter erheben nicht den Anspruch auf mathematische Genauigkeit, erreichen aber trotzdem einen wesentlich besseren Anschluß an die Meßdaten, als die von HoHEndorf errechneten Werte. In Tabelle 2 sind die erhaltenen Werte einander gegenübergestellt und die eingesetzten Parameterwerte gegeben.

Die Ansicht von Hohendorf, von den Ford-Walford-Parametern ausgehend auf relativ einfache Weise zu den mathematisch exakten Parametern der BerTalanfFyFunktion kommen zu können, ist nicht zutreffend. Sein Vorgehen führt zu Näherungswerten, liefert aber keine mathematisch einwandfreie Lösung. Sein Vorschlag für die Berechnung der WALFORD-Parameter erscheint dagegen nützlich, da man auf diesem Wege die Unschärfe, die allen Wachstumsdaten anhaftet, ausschalten und die errechneten Zahlen weiteren Auswertungen zugrunde legen kann. Bei der meist guten Annäherung der FORD-WALFORD-Werte an gegebene Meßreihen erscheint die hiermit gegebene Möglichkeit zur Ausgleichung statthaft.

Die Tatsache, daß die von HoHendorf vorgeschlagene Berechnung der BERTALANFFY-Parameter aus der WALFORD-Formel zu einem unbefriedigenden Ergebnis führte, zwang zu einer genaueren Durchleuchtung der mathematischen Grundlagen. Als 


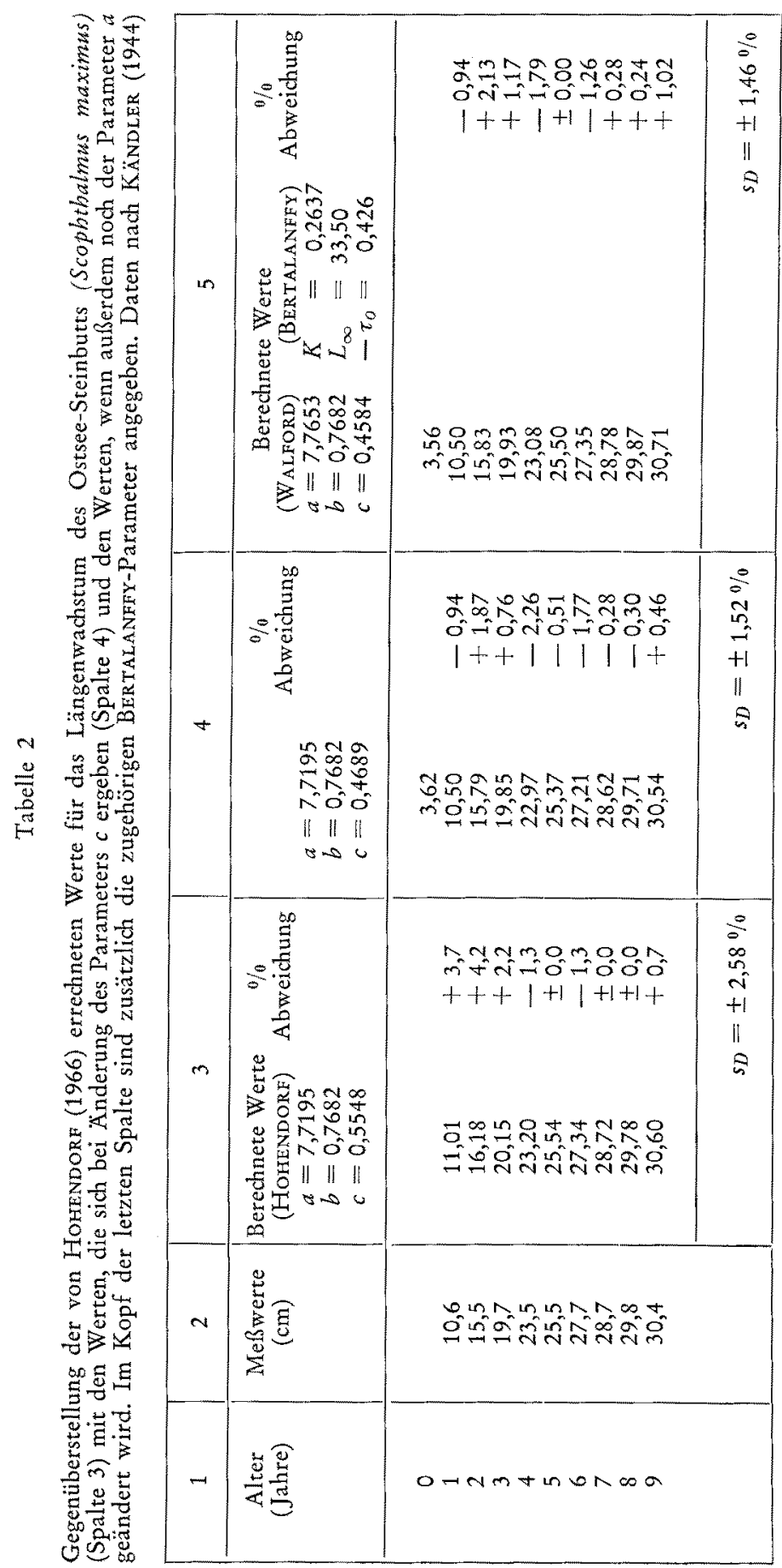


wesentlichste Erkenntnis ergab sich, daß die ForD-WALFORD-Funktion einen dritten Parameter enthält, den HoHENDorf ebensowenig erkannte, wie die früheren Autoren. Bei der üblichen Anwendung wird der dritte Parameter mit dem Anfangswert in die Berechnungen eingeführt.

\section{ABLEITUNG DER BERTALANFFY-FUNKTION AUS DER FORD-WALFORD-FORMEL}

Die Ford-Walford-Formel (Gleichung 1) ist die Gleichung einer Geraden, in der $b$ die Steigung wiedergibt und $a$ ihren Schnitt mit der Ordinate im Nullpunkt. $a$ ist aber nicht identisch mit $L_{0}$. Der Wert für $L_{0}$ ergibt sich zu:

$$
L_{0}=\frac{l_{1}-a}{b}
$$

Mit dem Wert 10,5 für $l_{1}$ und 0,7682 für $b$ und 7,7653 für $a$ wäre $L_{0}=3,56 \mathrm{~cm}$. Von $a$ ausgehend können wir zu $L_{0}$ kommen, wenn wir mit dem Faktor $c=0,4584$ multiplizieren.

Setzen wir den Parameter $c$ in die Ford-WALDORF-Formel ein, so erhalten wir:

$L_{0}=a \cdot c$

$l_{1}=a+a \cdot b \cdot c$

$l_{2}=a+a \cdot b+a b^{2} \cdot c$ etc.

$l_{n}=a \cdot\left(l+b+b^{2}+b^{3} \ldots \ldots+b^{n-1}\right)+a \cdot b^{n} \cdot c$

Die Klammer enthält die schon von FORD (1933) erkannte fallende geometrische Reihe von $b$ für $b<1$. Der dritte Parameter tritt nur im letzten Glied zusammen mit $a$ als Faktor auf, so daß wir es auch als $b^{\mathrm{n}} \cdot L_{0}$ formulieren können. Da der Wert von $b^{\text {n }}$ mit steigendem Exponenten immer geringer wird, vermindert sich damit auch immer mehr der Einfluß von $c$, beziehungsweise von $L_{\theta}$, auf die erreichte Größe und wird im Grenzfall zu 0.

Mathematisch sollte es möglich sein, aus der Differenz zwischen den Meßwerten und der ohne $c$ berechneten Reihe den Wert für $c$ zu berechnen. Die geringen Differenzen zwischen den beiden Reihen werden aber so stark durch die Unschärfe der Meßwerte beeinflußt, daß man kein befriedigendes Resultat erhält. Zunächst bleibt kein anderer Weg offen, als den in $l_{1}$ enthaltenen Parameter $L_{\theta}$ durch empirische Variation von $l_{1} \mathrm{zu}$ ermitteln, um den besten Anschluß an die übrigen Meßdaten zu erhalten. Im vorliegenden Fall habe ich $l_{1}$ in Stufen von $0,1 \mathrm{~cm}$ variiert. Die Summe der Quadrate der prozentualen Abweichungen ergab ein Minimum bei etwa 10,5. Dieser Wert liegt sehr nahe bei dem gemessenen Wert für $l_{1}$ und ergibt eine wesentlich bessere Näherung an die Meßdaten als der von Hohendorf errechnete Wert von 11,01. Die Erreichung einer höheren Genauigkeit erschien mir wenig sinnvoll, da auch die Meßwerte nur eine Stelle nach dem Komma angeben.

Durch den Umstand, daß für $n=\infty$ der Einfluß von $L_{0}$ gleich 0 wird, bleibt die Formulierung von TAYLOR gültig.

$$
L_{\infty}=\frac{a}{1-b}
$$


BERTALANFFY-Funktion und FORD-WALFORD-Formel

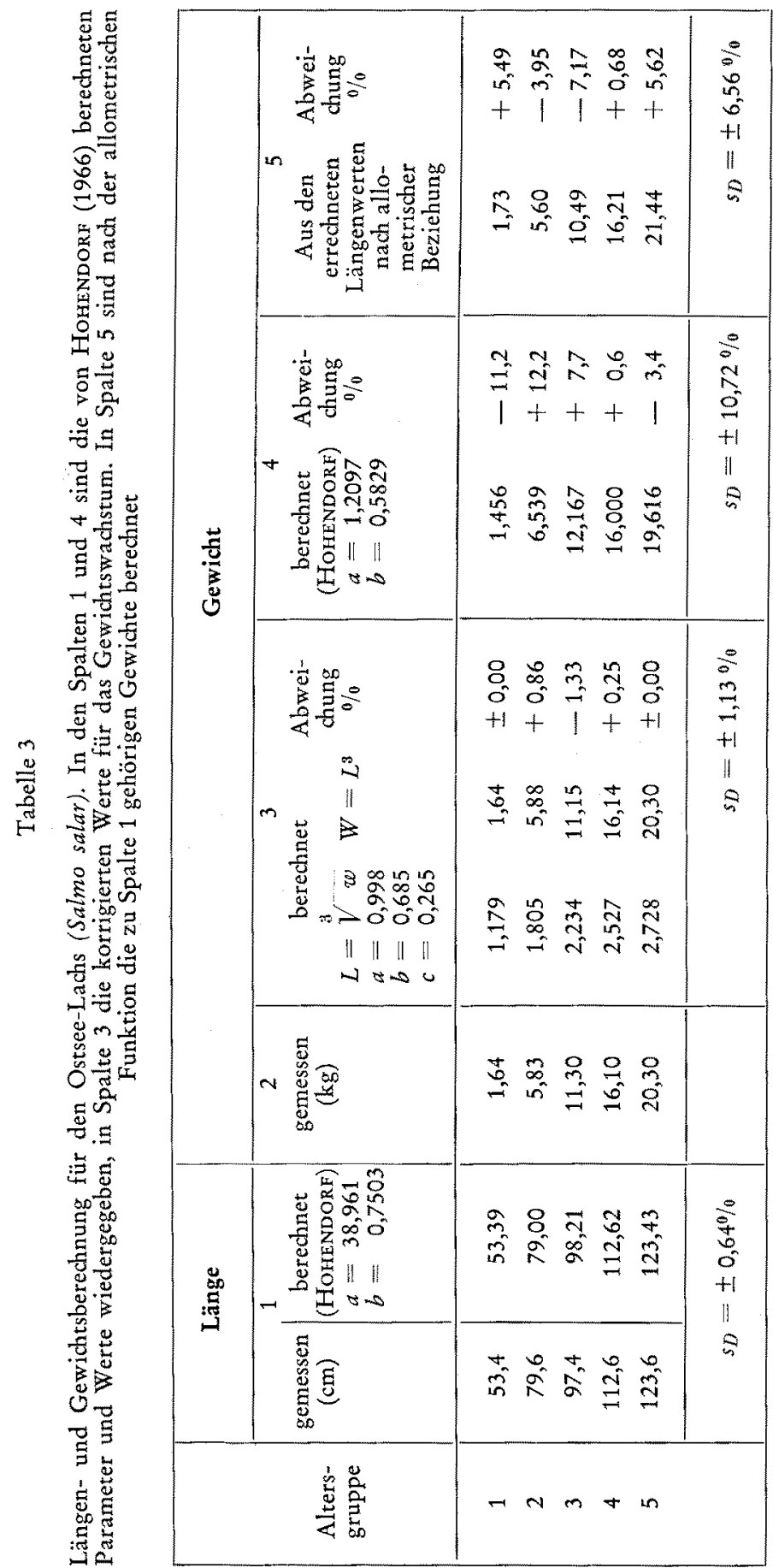


(Nicht zutreffend ist aber die früher von FORD und WALFORD und neuerdings wieder von BüCrManN (1967) aufgestellte Beziehung $L_{\infty}=\frac{l_{1}}{1-b}$ )

Mit der Einführung des dritten Parameters ergibt sich die Möglichkeit einer einfachen und übersichtlichen Ableitung der BERTALANpFy-Funktion aus der Ford-WALFORD-Formel. Als Ausgang wählen wir die Formulierung der Gleichung (7). Da $n$ der Anzahl der zugrunde gelegten Perioden entspricht, setzen wir an seine Stelle $\tau$ und außerdem an Stelle der Klammer die Summenformel für die geometrische Reihe von $b$ :

$$
l_{\tau}=a \cdot \frac{1-b^{\tau}}{1-b}+b^{\tau} \cdot L_{0}
$$

an Stelle von $\frac{a}{1-b}$ können wir nach Gleichung (3) $L_{\infty}$ setzen und erhalten dann in Ubereinstimmung mit der BertalanfFy-Funktion den Bezug auf die Maximalgröße:

$$
l \tau=L_{\infty} \cdot\left(1-b^{r}\right)+b^{\tau} \cdot L_{0}
$$

Auflösung der Klammer gibt:

$$
\begin{aligned}
I_{\tau} & =L_{\infty}-L_{\infty} \cdot b^{\tau}+b^{\tau} \cdot L_{\theta} \\
& =L_{\infty}-b^{\tau}\left(L_{\infty}-L_{\theta}\right)
\end{aligned}
$$

Das ist aber nichts anderes als die ursprüngliche Form der BERTALANFFY-Funktion, wenn wir $e^{-K r}$ entsprechend TAYLoR Gleichung (4) an Stelle von $b^{r}$ setzen. Diese einfache Ableitung macht noch einmal deutlich, daß die ForD-WALFORD-Formel drei Parameter enthält, wobei $L_{0}$ oder auch $L_{l}$ als Parameter fungiert. Sein Wert beeinflußt entscheidend die Ergebnisse und muß daher möglichst exakt bestimmt werden.

Da Hohendorf nicht erkannte, daß auch die Walford-Funktion drei Parameter enthält, hatte er auch sein Vergleichsmaß $s_{D}$ nicht richtig formuliert, wenn er bei dessen Berechnung nur zwei Freiheitsgrade abzieht. Auch bei der WALFord-Formel müssen - ebenso wie bei der BERTALANFFY-Funktion oder meinem eigenen Vorschlag - bei der Berechnung der Standardabweichung drei Freiheitsgrade berücksichtigt werden.

Wenn schon bei der relativ einfachen Längenberechnung die Parameter von HoHENDORF nicht $\mathrm{zu}$ wirklich befriedigenden Ergebnissen führen, so gilt das in besonderem Maße bei der von ihm durchgeführten Gewichtsberechnung. Da in diesem Falle die Resultate in der dritten Potenz in das Endergebnis eingehen, machen sich kleinste Abweichungen überaus stark bemerkbar.

HoHENDORF demonstriert sein Verfahren am Beispiel des Ostsee-Lachses (Salmo salar) (Tab. 3). Beim Längenwachstum führen die von ihm errechneten Parameter zu sehr guten Werten. Benutzt man sie, um auf dem Weg über die allometrische LängenGewichts-Beziehung die zugehörigen Gewichte zu berechnen, so kommt man zu einer nennenswert besseren Annäherung an die gegebenen Daten, als sie Hohendorf erreichte. Aus diesem Grund habe ich dieses Beispiel noch einmal durchgerechnet. Hierbei erhielt ich allerdings auch andere Parameterwerte für die Gewichtslängen als HoHENDORF einsetzte. Die graphische Kontrolle bestätigte die Richtigkeit der von mir benutzten WALFORD-Parameter, die in Tabelle 3 angegeben sind. Es war zu erwarten, daß in diesem Falle der dritte Parameter nicht unberücksichtigt gelassen werden darf, da $L_{\theta}$ sehr stark von $a$ differiert. Graphisch wurde für $L_{\theta}$ ein Näherungswert von 0,3 ge- 
funden. Durch Variation von $L_{\theta}$ ergab sich ein erstaunlich guter Anschluß an die dritten Wurzeln der Gewichte mit $L_{0}=0,265$. Da in diesem Falle a nahezu gleich 1,000 ist, sind in diesem Falle $c$ und $L_{\theta}$ praktisch identisch. Die aus diesen drei Parametern errechneten Gewichtslängen ergeben auch bei den Gewichten kaum Differenzen gegenüber den Meßdaten. Die Auswertung von HoHendorf ergab demgegenüber sehr starke Abweichungen (Tab. 3). Auf die Bestimmung der BerTALANFFy-Parameter habe ich in diesem Fall verzichtet, sie dürften eine ähnlich gute Näherung liefern. Offensichtlich ist auf dem schon von BERTALANFFY (1934) vorgeschlagenen Weg auch eine sehr gute Wiedergabe von Gewichtsdaten möglich.

Berechnet man aus der Gewichts-Längen-Beziehung das Maximalgewicht des Ostseelachses, so ergibt sich hierfür als Näherungswert $31,64 \mathrm{~kg}$. Nimmt man dagegen als Ausgangspunkt die Maximalgröße des Längenwachstums, so erhält man auf dem Wege über die allometrische Funktion ein Maximalgewicht von 43,68 kg. Die beiden Wege zur Berechnung des Maximalgewichtes weichen also sehr stark auseinander. Auch die anderen Parameter der Längen- und Gewichts-Funktionen lassen keine klaren mathematischen Beziehungen erkennen, worauf schon HoHENDORF hinwies. Hier liegt ein deutlicher Vorzug bei der von mir vorgeschlagenen Funktion (KRÜGER 1965), in welche die allometrischen Parameter eingesetzt werden können.

\section{DISKUSSION}

Die Ausführungen des letzten Abschnittes haben gezeigt, daß mit der Einführung des dritten Parameters $L_{0}(=a \cdot c)$ der Zusammenhang zwischen der Ford-WaLfordFormel und der BertalanfFy-Funktion sich sehr einfach demonstrieren läßt. Hierbei wird auch der von TAYLOR formulierte Zusammenhang der Parameter beider Funktionen klar ersichtlich. Trotzdem komplizieren einige Umstände die mathematische Beziehung zwischen den Parametern beider Funktionen.

Die erste Komplikation ergibt sich durch das Vorhandensein des dritten Parameters. Bei der üblichen Anwendung der FORD-WALford-Formel ist er in dem Anfangswert $l_{1}$ enthalten und wird durch ihn in die Berechnungen eingeführt. Nun versucht Hohendorf auf dem Umweg über die Bertalanffy-Funktion den Wert von $l_{1}$ zu berechnen. Hierbei begeht er aber den Fehler, daß ex in seiner Ableitung (seine Gleichung 4) für $l_{1}$ den Parameter $a$ der Walford-Funktion in die BerTalanfFYFunktion einsetzt. Auf diesem Umweg kommt er dann zu einem zwar von $a$ abweichenden Wert für $l_{l}$, der aber in keiner Weise optimal ist. Er kann es schon aus dem Grunde nicht sein, da seine WALFORD-Parameter nur für die der Berechnung zugrunde gelegten Wachstumsreihe optimal sind, nicht aber für eine Reihe mit einem abweichenden Anfangswert. Auf diese Weise ändert er den Wert des dritten Parameters $c$, der in funktioneller Beziehung zu $l_{1}$ steht.

Die theoretisch gegebene Möglichkeit, den Wert für $c$ aus den Meßdaten zu berechnen, scheitert an ihrer unvermeidlichen Unschärfe. So bleibt nur die Möglichkeit, rein empirisch durch Einsetzen verschiedener Werte für $l_{1}$ den Wert zu ermitteln, der die geringsten quadratischen Abweichungen von den Meßwerten liefert. Hierdurch hat man bei gegebener Steigung $b$ einen Näherungswert für $l_{1}$ gefunden und kann von 
ihm ausgehend den Näherungswert für $L_{\theta}=a \cdot c$ berechnen. Nun variiert man $a$, um gegebenenfalls eine noch bessere Annäherung an die Meßdaten zu erreichen. Auf diesem Wege konnte die mittlere quadratische Abweichung von den Daten des gegebenen Beispiels fast auf die Hälfte gegenüber der Auswertung von HoHENDorf reduziert werden. Von den von HoHendorf berechneten Parametern habe ich nur den Wert für $b$ übernommen. Ob er optimal ist, konnte ich aus technischen Gründen nicht mehr prïfen.

Noch ein anderes Versehen ist HoHENDorf unterlaufen. Die Ford-WaLford-Formel stellt eine lineare Funktion dar, und die von ihm angewandte Regressionsberechnung liefert daher die Parameter für eine minimale lineare Abweichung von den Meßwerten. Die Güte der Wiedergabe prüft er aber an der prozentualen Abweichung. Die Regressionsgerade für die relativen Abweichungen fällt aber nicht mit der für die linearen Abweichungen zusammen. In dieser Hinsicht sind logarithmische Funktionen günstiger. Die WALFORD-Formel richtet sich vor allem nach den Werten der höheren Größenklassen, während bei der relativen Beurteilung die Wiedergabe der kleinen Größenklassen entscheidend ist. Es spricht für die sehr exakte Steuerung des Wachstumsvorganges, wenn auch bei einer linearen Formulierung die errechneten Zahlen in so guter Näherung den Wachstumskurven folgen. Solange aber keine mathematische Lösung gefunden ist, die bei der FORD-WALFORD-Formel die relativen Abweichungen zum Minimum macht, kann man ihre Parameter nur als Näherungslösung für die Parameter der BertalanfFy-Funktion betrachten, von denen ausgehend die exakten Parameter nur empirisch durch iterative Berechnung gefunden werden können.

Wir sahen, daß ein rein rechnerisches Vorgehen an verschiedenen Punkten kein befriedigendes Resultat lieferte. Die stets gegebenen Abweichungen biologischer Meßwerte von den mathematisch exakten Werten lassen nur bei der erforderlichen Kritik rein formale Berechnungen zu. Die vom theoretischen Kurvenverlauf abweichenden Meßwerte beeinflussen die Berechnung in schwer kontrollierbarer Weise und gestatten daher zumeist nur die Gewinnung von Näherungswerten. Von ihnen ausgehend kann man dann aber schrittweise durch systematische Anderung der Parameter zu Werten kommen, die einen besseren Anschluß an gegebene Daten liefern.

Die Biomathematik erfordert für brauchbare Lösungen im allgemeinen die Anwendung von Näherungsverfahren. Das bedingt eine häufige Wiederholung recht komplizierter Rechenoperationen und erfordert daher einen großen Zeitaufwand. Die modernen Elektronenrechner sind unentbehrliche Hilfsmittel, um biomathematische Probleme anzugreifen und zu lösen. Computer-Programme sind für die BertalanffyFunktion zum Beispiel von FABENS (1965) und RADWAY (1966) entwickelt worden. Es wäre günstig gewesen, wenn HoHENDORF sie zur Kontrolle seines Verfahrens herangezogen hätte.

Die grundsätzliche Möglichkeit, tierische Wachstumsdaten durch ein mathematisches Modell zu reproduzieren, kann heute nicht mehr übersehen und bestritten werden, nachdem mehrere praktisch gleich leistungsfähige Lösungen vorliegen.

In der vorliegenden Untersuchung konnte in sehr einfacher und übersichtlicher Weise die Identität von BERTALANFFY-Funktion und WALFORD-Formel bestätigt werden. Ich selbst habe eine neue Wachstumsfunktion vorgeschlagen (KRÜGRR 1965), die offensichtlich in weitem Bereich der BERTALANFFY-Funktion parallel verläuft. Auch 
hinsichtlich der rechnerischen Werte kommt sie in der Güte der Wiedergabe von Wachstumsdaten der BerTALANFFY-Funktion zum mindesten gleich. Hierauf wies ich schon früher hin, und Horendorf bestätigte diese Parallele. Mathematisch beruht sie aber auf grundsätzlich anderer Basis.

Es liegen also jetzt zumindest zwei Funktionen vor, die für praktische Zwecke zur Wiedergabe des Fischwachstums geeignet erscheinen. Nicht berücksichtigen will ich hier die ebenfalls oft zitierte GOMPERTz-Funktion. Sie wurde nur relativ selten angewandt, da bei ihr die Parameterbestimmung und damit auch ihre Beurteilung schwierig ist. Die Entscheidung über die Eignung einer Wachstumsfunktion liegt also nicht mehr bei der Güte der Wiedergabe von Meßreihen, sondern bei ihren mathematischen Eigenschaften und dem Aussagewert der einzusetzenden Parameter.

Mein Vorschlag besitzt vor der BerTALANFFy-Funktion zwei wesentliche Vorzüge. Zum ersten schließt sie auch für die Längenwerte einen. Wendepunkt ein, wie er theoretisch zu erwarten ist. Noch wesentlicher ist der zweite Vorzug, daß nämlich ihre Parameter in mathematisch festgelegter Beziehung zur allometrischen Funktion stehen. Hierdurch gestattet die neue Funktion auch eine direkte Auswertung von Gewichtsdaten, ohne daß man den Umweg über die Gewichtslänge $(\sqrt[3]{w})$ einschlagen muß. Noch entscheidender wird dieser Vorzug bei der Bearbeitung physiologischer Probleme (KRÜGER 1967).

Die Bertalanffy-Funktion und mein eigener Vorschlag:

$$
y_{\mathrm{x}}=\frac{y_{\max }}{N^{\frac{1}{x+\xi}}}
$$

zeigen mathematisch einen sehr ähnlichen Aufbau. Beide enthalten eine Maximalgröße als Parameter, welcher sich der Organismus bei unbegrenztem Wachstum nähert, ferner einen Geschwindigkeitsparameter, der die Steigung der Wachstumskurve wiedergibt und einen additiven Zeitwert $\tau_{0}$ bzw. $\xi$. Ein direkter Vergleich der Parameter ist allerdings nicht möglich. Der Maximalwert liegt bei meiner Funktion wesentlich höher als bei der BerTALANFFY-Funktion. Durch den größeren Abstand von den Meßdaten läuft er aber nicht so leicht Gefahr, unterhalb von real vorkommenden Maximalgrößen zu liegen, wie es HohENDOrf für den Nordseesteinbutt angibt.

Alle Parameter, die wir bei unseren Berechnungen einsetzen, stellen zunächst rein mathematische Zahlenwerte dar, und es erscheint mir wenig sinnvoll, ihre biologische Deutung zu stark zu bewerten. Ihre biologische Interpretation bleibt eine sekundäre Aufgabe.

Den Geschwindigkeitsparameter stellt in der BertalanfFy-Funktion der Exponent $K$ dar. Seine Beziehung zur Wachstumsgeschwindigkeit ist aber nicht so einfach, wie es Hohendorf (1966) annimmt. Das zeigt sein Beispiel für das Längenwachstum des Nordseebutts (Scophthalmus maximus) (seine Tabelle 5).

Er findet für die Männchen des Nordseebutts für $K$ den Wert 0,2690 und für die Weibchen 0,2594. Demnach würden die Männchen schneller wachsen als die Weibchen. In Wirklichkeit wachsen letztere in dem beobachteten Zeitraum von $8,3 \mathrm{~cm}$ auf $60,82 \mathrm{~cm}$ heran, die Männchen dagegen nur auf 53,5 cm. Das Wachstum der Weibchen 
ist also eindeutig stärker. Die Ursache für diesen Widerspruch liegt darin, daß $K$ die Geschwindigkeit bestimmt, mit der sich das Wachstum dem Endpunkt nähert. In den Wert für $K$ geht die Maximalgröße ein, und diese ist bei beiden Geschlechtern sehr unterschiedlich. Sie beträgt nach HOHENDORF für die Männchen $53,88 \mathrm{~cm}$ und für die Weibchen $62,17 \mathrm{~cm}$. Da man beim Wachstumsvergleich auf der Basis der BERTALANFFYFunktion den Wert für $L_{\infty}$ bei verschiedenen Arten oder Geschlechtern nicht konstant halten kann, bildet $K$ keinen eindeutigen Ausdruck für die Wachstumsgeschwindigkeit. Der $b$-Wert der FORD-WALFORD-Formel bietet in dieser Beziehung eine günstigere Vergleichsbasis, da er vom Anfangswert $l_{t}$ ausgeht, der bei beiden Geschlechtern gleich ist. Der $b$-Wert beträgt in diesem. Falle für die Männchen 0,7642 und für die Weibchen 0,7715 . Er gibt das Verhältnis der Wachstumsgeschwindigkeit also besser wieder.

Während die Geschwindigkeitsparameter der BERTALANFFy-Funktion und der WALFORD-Funktion einen exakten Vergleich zulassen, ist ein solcher nicht mit dem Geschwindigkeitsparameter meiner Funktion möglich, da hier die Wachstumsgeschwindigkeit durch zwei Parameter ausgedrückt wird: $\log N$ und $\xi$.

Der dritte Parameter, den die BertalanfFy-Funktion und mein eigener Vorschlag enthalten, ist mathematisch gesehen die Zeitspanne, während der sich der Organismus von der Dimension 0 zu der ersten gemessenen Länge entwickelt hat. Da aber Larven- und Embryonalentwicklung offensichtlich bei beiden Funktionen nicht von den Parametern des postlarvalen Wachstums erfaßt werden, stellt dieses „pränatale" Alter einen rein rechnerischen Wert dar, der von der mathematischen Interpretation der Wachstumskurve bestimmt wird. Dadurch, daß die Bertalanffy-Funktion keinen Wendepunkt enthält, fällt sie in dem pränatalen Bereich wesentlich stärker $a b$, und es ergeben sich hierdurch geringere Werte für das pränatale Alter, als bei meiner Funktion. Die $\tau_{0}$-Werte liegen daher eher in der Dimension des pränatalen Alters, aber auch HoHENDORF ist in dieser Deutung sehr zurückhaltend.

Die Bedeutung des $\xi$-Wertes meiner Funktion liegt vor allem darin, daß er die Krümmung der relativen Wachstumskurve kennzeichnet, er ist mathematisch also ein Krümmungsparameter. Sein Vorzug ist ferner, daß er wenig kritisch ist und bei schneller wachsenden Fischen in vergleichbarer Größenordnung liegt. Hierdurch wird ein Vergleich von Wachstumsgeschwindigkeiten durch den Geschwindigkeitsparameter erleichtert. An anderer Stelle werde ich darüber berichten. Bei der Bertalanffy-Funktion ist $\tau_{\theta}$ sehr kritisch und die Beziehung zur Kurvenkrümmung nicht so eindeutig.

Die Parameter der FORD-WALFORD-Funktion bleiben in ihrer mathematischen Deutung noch näher zu analysieren. Anderungen des Parameters $c$ bedingen eine Verschiebung der Werte auf der Regressionsgeraden, während Anderungen des Wertes von a die Regressionsgerade parallel zu sich verschieben. In welcher Weise die Wachstumskurven hierdurch beeinflußt werden, ist nicht ohne weiteres zu übersehen.

Meine Ausführungen und mathematischen Begründungen haben gezeigt, daß der an sich gegebene Zusammenhang zwischen den Parametern der BertalanfFY-Funktion und der FORD-WALFORD-Formel nicht in so einfacher Weise für die Bestimmung der BERTALANFFY-Parameter ausgewertet werden kann, wie es HOHENDORF vorschlägt.

Wenn HoHENDORF (1966) in seiner Zusammenfassung behauptet, „durch die Verwendung dieser linearen Beziehung als Rekursionsformel für die BERTALANFFY-Funktion kann ein einfaches und leicht anwendbares Verfahren zur exakten Bestimmung 
der Parameterwerte und Wachstumswerte auf rein rechnerischem Wege angegeben werden ", so konnte das nicht bestätigt werden. Zur Klärung des Zusammenhangs zwischen der BERTALANFFY-Funktion und meinem eigenen Vorschlag war ich an einer wirklich exakten Bestimmung der BerTALANFFY-Parameter interessiert. Leider erfüllt der Vorschlag von HoHeNdorf nicht diese Forderung. Wenn man den auf mechanischen Tischrechnern mühsamen Weg iterativer Berechnungen vermeiden will, bleibt wenigstens vorläufig - die graphische Lösung, wie ich sie zur Ermittlung von $L_{\infty}$ anwandte, ein wichtiges Hilfsmittel. Daneben erleichtert die Ausnutzung der Beziehungen zur Ford-Walford-Funktion die Parameterberechnungen. Ich hielt es für wichtig, meine Erfahrungen mit dem Vorschlag von HoHendorf zu veröffentlichen, um künftige Benutzer über die Grenzen dieses Verfahrens und seine Anwendbarkeit zu unterrichten. Vollkommen ungeeignet ist sein Verfahren zur Berechnung von $l_{1}$. Mathematisch richtig ist nur der eingegebene Anfangswert. Für eine bessere prozentuale Annäherung muß der optimale Wert für $L_{\theta}$ beziehungsweise $c$ als dritter Parameter bestimmt und angegeben werden.

Die Bearbeitung biomathematischer Fragen deckt immer wieder bei den verschiedensten Autoren fehlerhafte Formulierungen auf. Nur mit dem Bemühen, auf mathematisch einwandfreier Basis rechnerisch einen möglichst guten Anschluß an gegebene Meßdaten zu erreichen, wird es möglich sein, mathematische Verfahren bei der Erforschung der Lebenserscheinungen einzusetzen. Von groben Näherungen können wir keinen Fortschritt unserer Erkenntnis erwarten.

\section{ZUSAMMENFASSUNG}

1. Zur Charakterisierung des Wachstums von Fischen hat HoHendorf (1966) versucht, die von TAYLOR formulierte Beziehung zwischen den Parametern der BER-

TALANFFY-Funktion und der FORD-WALFORD-Formel: $L_{\infty}=\frac{a}{1-b}$ und $K=-\ln b$ zur Berechnung der Bertalanffy-Parameter auszunutzen. An Hand der mathematischen Bearbeitung einiger Beispiele wird gezeigt, daß sein Verfahren nicht $z u$ Parametern führt, die als exakt $z u$ bezeichnen sind.

2. Die Ford-Walford-Formel enthält einen dritten Parameter $c$, der mit $l_{1}$ in die Berechnung eingeführt wird.

3. Der dritte Parameter gestattet in sehr einfacher Weise die Ableitung der BERTALANFFY-Funktion von der FORD-WALFORD-Formel und bestätigt die von TAYLOR aufgestellte Beziehung $z$ wischen den Parametern.

4. Es werden die Gründe untersucht, aus denen sich bei Anwendung des Verfahrens von HoHENDORF nur Näherungswerte ergeben.

5. Hierbei ist die Tatsache entscheidend, daß die Parameter der Regressionsgeraden der FORD-WALFORD-Formel die linearen Differenzen zwischen den berechneten und den gemessenen Werten zum Minimum machen, während bei der Wachstumsberechnung das Minimum der prozentualen Abweichungen gesucht wird. 


\section{ZITIERTE LITERATUR}

BertalanfFy, L. von, 1934. Untersuchungen über die Gesetzlichkeit des Wachstums. 1. Allgemeine Grundlagen der Theorie. Arch. EntwMech. Org. 131, 613-653.

Beverton, R. J. \& Hol', S. J., 1957. On the dynamics of exploited fish populations. Fishery Invest., Lond. (Ser. 2) 19, 1-533.

Bückmann, A., 1963. Das Problem der optimalen Befischung. Arch. FischWiss. 14 (Beih. 1), 1-107.

Deržavin, A. N., 1922. [Sevrjuga (Acipenser stellatus).] - Der gestirnte Stör. - [Der Scherg. Biologičeskij očerk. - Eine biologische Studie. (Russ.) Izv. bakin. ichtiol. Lab. 1. (Zit. nach Schmalyausen, J., 1928.)]

Fabens, A. J., 1965. Properties and fitting of the Bertalanffy growth curve. Growth 29, 265-289.

ForD, E., 1933. An account of the herrings investigations conducted at Plymouth during the years from 1924-1933. J. mar. biol. Ass. U. K. 19, 305-384.

Gulland, J. A. \& Holt, S. J., 1960. Estimation of growth parameters for data at unequal time intervals. J. Cons. perm. int. Explor. Mer 25, 47-49.

Hohenoorf, K., 1966. Eine Diskussion der Bertalanfy-Funktion und ihre Anwendung zur Charakterisierung des Wachstums von Fischen. Kieler Meeresforsch. 22, 70-97.

Kändler, R, 1944. Über den Steinbutt der Ostsee. Ber. dt. wiss. Kommn Meeresforsch. 11, 73-136.

KRÜGER, F., 1965. Zur Mathematik des tierischen Wachstums. 1. Helgoländer wiss. Meeresunters. 12, 78-136.

- 1967. Theoretische Physiologie: Wachstumsmathematik. Jber. Biol. Anst. Helgoland 1966, 24-25.

RADWAY, A. K., 1966. A method of fitting growth curves of the Bertalanffy type to observed data. J. Fish. Res. Bd Can. 23, 163-179.

TAYLOR, C. C., 1958. A note on Lee's phenomenon in Georges Bank haddock. Spec. Publs int. Commn NW Atlant. Fish. 1, 243-251.

Schmalhausen, J., 1928. Das Wachstumsgesetz und die Methode der Bestimmung der Wachstumskonstante. Arch. EntwMech. Org. 113, 462--519.

WALFORD, L. A., 1946. A new method of describing the growth of animals. Biol. Bull. mar. biol. Lab., Woods Hole 90, 141-147. 